\title{
Embodied Communication in Humans and Machines
}

\author{
Ipke Wachsmuth and Günther Knoblich
}

Abstract. The challenge to develop an integrated perspective of embodiment in communication is taken up by an international research group hosted by Bielefeld University's Center for Interdisciplinary Research (ZiF-Zentrum für interdisziplinäre Forschung) from October, 2005 through September, 2006. An international conference was held there on 12-15 January, 2005 to define a research agenda that will explicitly address embodied communication in humans and machines.

Embodiment has become one of the most promising paradigms in cognitive science and a challenge to AI research. In an invitational conference hosted by the Center for Interdisciplinary Research (ZiF) at Bielefeld University in Germany, 24 highly acclaimed speakers from various disciplines presented their perspectives pertaining to conceptual issues of embodiment; the phylo- and ontogenesis of communication; bodily gestures; understanding and communicating intentions, emotions, and symbols; and the role of bodily action in language and speech. The talks were centered around the two aims of the research group, namely, (1) to obtain a more profound understanding of human communication and its evolution, and (2) to study machine communication both as a means of modeling human communicative abilities and of advancing the human-technology interface.

Gestures were a central topic of the conference. Primatologist Josep Call (Max Planck Institute of Evolutionary Anthropology, Leipzig, Germany) reported of the rich and flexible gesture repertoire of non-human primates. Psychologist Bennett Bertenthal (University of Chicago, USA), and psycholinguists Sotaro Kita (University of Bristol, UK) and Susan Duncan (University of Chicago, USA) pointed out how gestures form an intimate connection with human speech. Moreover, gestures are responsive to the gestures of others, coupling speakers and listeners in a close "social loop" in which a real-time processing of bodily signals seems at least as important as semantic analysis of words and sentences.

The question how the structure of bodily communication could be captured in rule systems was raised by communication psychologist Isabella Poggi (Università Roma Tre, Rome, Italy) who presented ongoing research on mode-specific lexicons, such as "gestionaries", "gazeionaries" and "touchionaries", as an equivalent of dictionaries in spoken language. How these ideas could be integrated in human-machine interaction was addressed by computer scientist Catherine Pelachaud (Université de Paris 8, Montreuil, France). She showed how Affective Presentation Markup Language (APML) markers are converted into facial signals to provide embodied conversational agents with multimodal synchronized behavior, including gestures, adaptation to context, and facial display. 
As a glance to the future of human-computer interaction, two virtual creatures were around at the conference. Computer scientist Kristinn Thórisson (Reykjavík University, Iceland) demonstrated Gandalf, a virtual agent guide to our galaxy. Gandalf is able to rotate and zoom projected images of planets at the user's command (uttered verbally or by gesture) and answer questions about the planets. In Bielefeld University's virtual reality laboratory, the conference participants met the "multimodal assembly expert (MAX)." Developed by the Bielefeld AI group, Max is able to imitate human gestures and exhibit human-like synthetic speech and coverbal gesture while constructing an airplane from a construction kit in cooperation with a human partner.

Imitation was described as a form of embodied communication by computer scientist Aude Billard (Ecole Polytechnique Lausanne, Switzerland). In her view, imitation is of great relevance in robot learning, namely, to find a way in which humans and robots could learn a common means of communication. Computer scientist Luc Steels (Free University, Brussels, Belgium and Sony Computer Science Laboratory, Paris) showed that comparably simple systems, linked to a surrounding world environment by a camera, are able not only to achieve agreement on the meaning of concepts but also to develop simple forms of syntax, without being equipped with explicit rules of communication.

Despite its theoretical importance, the concept of embodiment is far from clear. Does a body need flesh and blood?, linguist Jens Allwood (University of Göteborg, Sweden) asked, or might wire and metal be sufficient, or a simulation in virtual reality? When apes can learn to control a robot arm through an electrode implanted in their brains, philosopher Joëlle Proust (Institut JeanNicod, Paris, France) added, then where are the borders of one's own body? Psychologist Lawrence Barsalou (Emory University, Atlanta, GA, USA) explained how abstract symbols are presumably grounded in modality-specific brain systems and how categorical knowledge may be a form of reenactment or simulation. Theoretical biologist Holk Cruse (Bielefeld University, Germany) put forward the idea that the brain might generally use its model of the body to simulate future states resulting from action, that is, plan ahead.

By the end of the conference, a long list of challenging questions was identified. How are bodily and verbal communication related? What is the role of the body in concept formation? What is the evolutionary relation between embodied communication and theory of mind? Are there specific control structures in embodied communication? How are different levels integrated in these? How can they be integrated in AI systems? What is the role of the environment? These and more questions are on the agenda for the $\mathrm{ZiF}$ research year on embodied communication, which will have its kick-off with an opening conference in October, 2005. Details are available at the center's Web site. ${ }^{1}$

\section{Note}

1. http://www.uni-bielefeld.de/ZIF/FG/2005Communication/. 


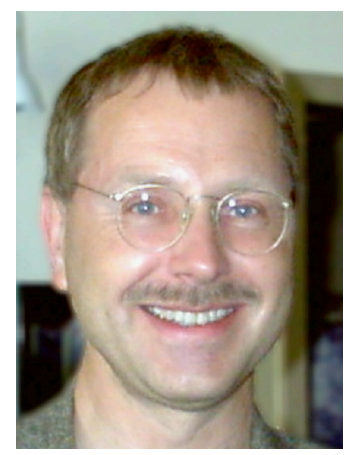

Ipke Wachsmuth is director of the Center for Interdisciplinary Research (ZiF) and chair of artificial intelligence at the University of Bielefeld, Germany. He holds a mathematics Master's degree and a Ph.D. both from the University of Hannover, Germany, and an informatics Habilitation degree from the University of Osnabrück, Germany. A major part of his current research is related to the Collaborative Research Center "Situated Artificial Communicators" where he conducts joint projects with linguists and psycholinguists on situated language and coverbal gesture. Practical aspects of his research aim at advanced human-machine interfaces which realize embodied communication in fully immersive virtual reality.

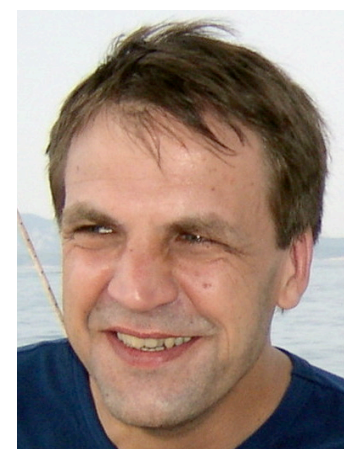

Günther Knoblich is an associate professor for cognitive psychology and neuroscience at Rutgers University, Newark. Previously he was a research scientist at the Max Planck Institute for Psychological Research, Munich, Germany (1997-2004) and a visiting scientist at the University of Illinois at Chicago (1995-1996). He received a Habilitation degree (2004) from the University of Munich and a Ph.D. in psychology (1997) from the University of Hamburg, Germany. His current research interests include self and other in perception and action; joint action; action observation and action understanding; body perception; and insight problem solving. 